\title{
Prevalência de excesso de peso e obesidade em pessoas com transtorno do espectro autista: uma revisão bibliográfica
}

\author{
CAROLINA LOURENÇO REIS QUEDAS \\ Universidade Anhanguera, Osasco, SP, Brasil. \\ E-mail: carolinaquedas@gmail.com \\ EDUARDO HENRIQUE MENDES \\ Universidade Anhanguera, Osasco, SP, Brasil. \\ E-mail: mendeseduardo77@hotmail.com \\ TIAGO BARBOSA TOLEDO \\ Universidade Federal de São Paulo (Unifesp), São Paulo, SP, Brasil. \\ E-mail: tiagotoledo@yahoo.com.br
}

\section{Resumo}

A obesidade e o ganho excessivo de peso têm sido um problema de saúde pública em muitos países, e algumas pesquisam apontam que a obesidade é a segunda causa de mortes evitáveis nos Estados Unidos, perdendo apenas para o tabagismo. Segundo muitos estudos, a inatividade física, os déficits na interação social e o comportamento sedentário estão relacionados ao aumento do risco da obesidade e do ganho excessivo de peso em pessoas com transtorno do espectro autista. Este estudo teve como objetivos levantar e analisar artigos sobre a prevalência e os fatores que possam contribuir para a obesidade e ganhos de peso em pessoas com transtorno do espectro autista, por meio das bases de dados PubMed e Bireme. O método utilizado foi qualitativo-descritivo, por meio dos seguintes descritores específicos: autism and obesity e overweight and autism. Foram encontrados 396 artigos publicados entre 2018 e 2020. Desse total, cinco estavam de acordo com os critérios de inclusão deste estudo. A conclusão deste estudo foi que indivíduos com autismo têm maior risco de ter obesidade e ganho excessivo de peso em relação à população neurotípica, e várias causas listadas, como a inatividade física e a falta de conhecimento nutricional, são fatores impor- 
tantes para a instalação de quadro que pode causar problemas de doenças crônicas e perda de qualidade de vida.

\section{Palavras-chave}

Autismo. Obesidade. Transtorno do espectro autista. Excesso de peso. Inatividade física.

\section{INTRODUÇÃO}

O transtorno do espectro autista (TEA) é um dos transtornos do neurodesenvolvimento. Trata-se de uma condição que se manifesta nos primeiros anos de vida e que tem como critérios de diagnóstico os déficits na comunicação social e nos padrões repetitivos e restritos (AMERICAN PSYCHIATRIC ASSOCIATION, 2013).

De acordo com a Sociedade Brasileira de Endocrinologia e Metabologia (Sbem), a obesidade é caracterizada por acúmulo de gordura corporal no indivíduo, e, para o diagnóstico em adultos, o parâmetro mais utilizado é o índice de massa corporal (IMC), que é calculado dividindo o peso do indivíduo pela altura elevada ao quadrado. Segundo a Organização Mundial da Saúde (OMS), uma pessoa tem peso normal quando o IMC está entre 18,5 e 24,9, e, a partir de 30, o indivíduo é considerado obeso. Já o excesso de peso acontece quando o indivíduo tem maus hábitos alimentares, por questões genéticas ou por disfunções endócrinas, e o IMC fica entre 25,0 e 30,0.

Obesidade é uma síndrome multifatorial em que ocorrem alterações fisiológicas, bioquímicas, anatômicas e metabólicas. Além disso, há alterações psicológicas e sociais. Porém, existem fatores ambientais que podem provocar a obesidade, como consumo excessivo de alimentos sem a realização de atividades físicas (ABREU, 2011).

$\mathrm{Na}$ atualidade, sabe-se que crianças com TEA têm obesidade e sobrepeso com maior frequência, mas grande parte dos estudos é internacional, e existem poucos estudos nacionais que provam isso (KUMMER, 2016).

Muitas pesquisam apontam que jovens com TEA têm maior risco em ganhar peso excessivo e, consequentemente, ter obesidade. Muitos fatores estão relacionados com o ganho de peso e a obesidade em jovens com TEA, como déficits na comunicação social, influências biológicas, comorbidades secundárias, medicações, desafios em participar de programas de exercícios físicos e esportes, ambiente alimentar, falta de profissionais qualificados para desen- 
volver programas de exercícios físicos e alimentação inadequada (DHALIWAL et al., 2019; BLOCK, 2016).

Este estudo teve como objetivos levantar e analisar artigos que apresentam métodos de estudos voltados às possíveis causas do excesso de peso e da obesidade em indivíduos diagnosticados com TEA.

\section{MÉTODO}

Esta pesquisa é de cunho qualitativo-descritivo e foi estruturada em três etapas: 1. revisão bibliográfica realizada por meio de busca sistematizada nas bases de dados eletrônicos PubMed e Bireme; 2. levantamento de dados sobre obesidade e ganho excessivo de peso por indivíduos diagnosticados com TEA; 3. análise dos artigos, considerando objetivo, método e resultados.

Na primeira etapa, definiram-se os bancos de dados, nos quais se utilizaram os descritores obesity and autism spectrum disorders (ASD) e overweight and asd. Nas buscas, utilizou-se o filtro por palavras-chave no qual é possível realizar o cruzamento de palavras presentes em categorias específicas. Selecionaram-se apenas textos que mencionavam obesidade e sobrepeso em indivíduos com TEA, a partir de 2018, cujas temáticas estivessem de acordo com os objetivos do presente trabalho e disponíveis on-line.

Excluíram-se os trabalhos que não estavam voltados para obesidade e sobrepeso no TEA e/ou não citavam causas para essa condição.

Na segunda etapa, foram levantados dados dos artigos pertinentes ao tema obesidade e ganho de peso excessivo em indivíduos com TEA. Na terceira etapa, realizaram-se a leitura e a análise dos artigos. Essa busca sistemática foi realizada no período de 25 de junho a $1^{\circ}$ de julho de 2020 .

\section{RESULTADO E DISCUSSÃO}

No total, a busca resultou em 396 estudos, dos quais 391 foram excluídos após a leitura do título, dos resumos e dos trabalhos, conforme critérios de exclusão. O fluxograma da busca está ilustrado na Figura 1. Selecionaram-se ao final da pesquisa cinco artigos que atendiam às propostas dos autores para investigação dos trabalhos disponíveis na literatura voltados à temática obesidade e ganho de peso da pessoa com TEA. O Quadro 1 apresenta os objetivos, os métodos e as conclusões dos textos. 


\section{Figura 1 Fluxograma do estudo}

Total dos artigos encontrados

$$
N=396
$$

PubMed $(n=327)$

Bireme $(n=69)$

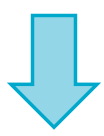

Artigos excluídos $(n=378)$

Verificação de textos livres $(n=184)$

Após leitura dos resumos $(n=185)$

Após leitura completa $(n=20)$

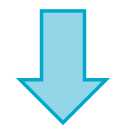

Artigos incluídos

PubMed $(n=2)$

Bireme $(n=3)$

Fonte: Elaborada pelos autores. 


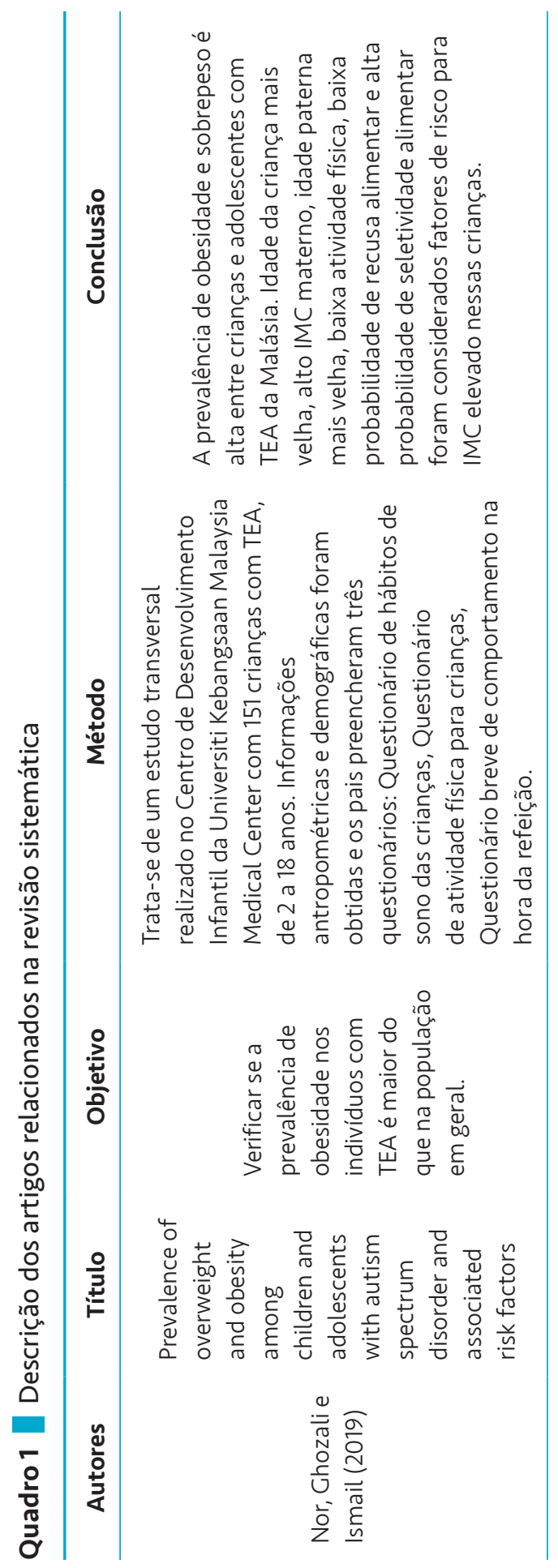




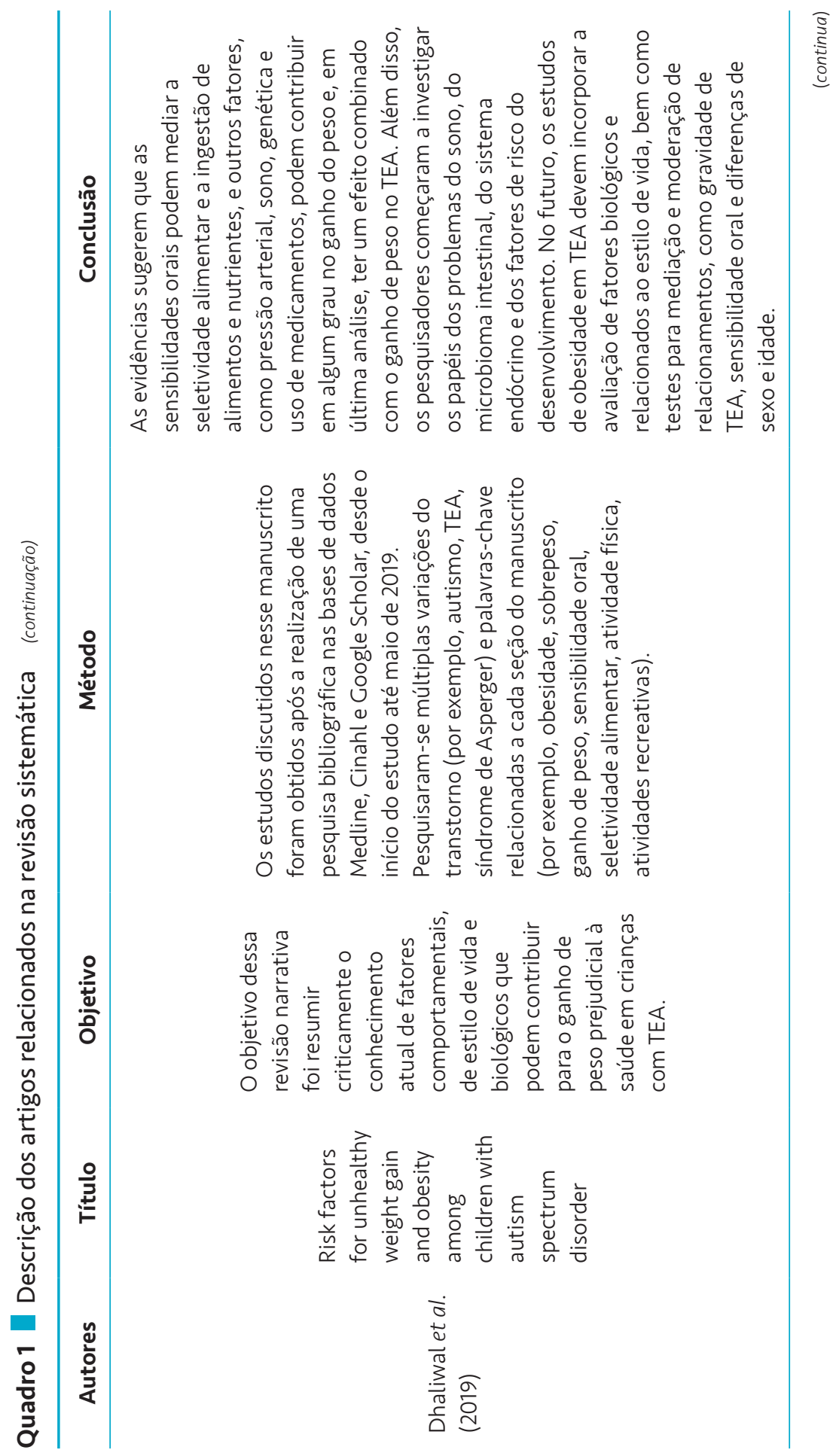




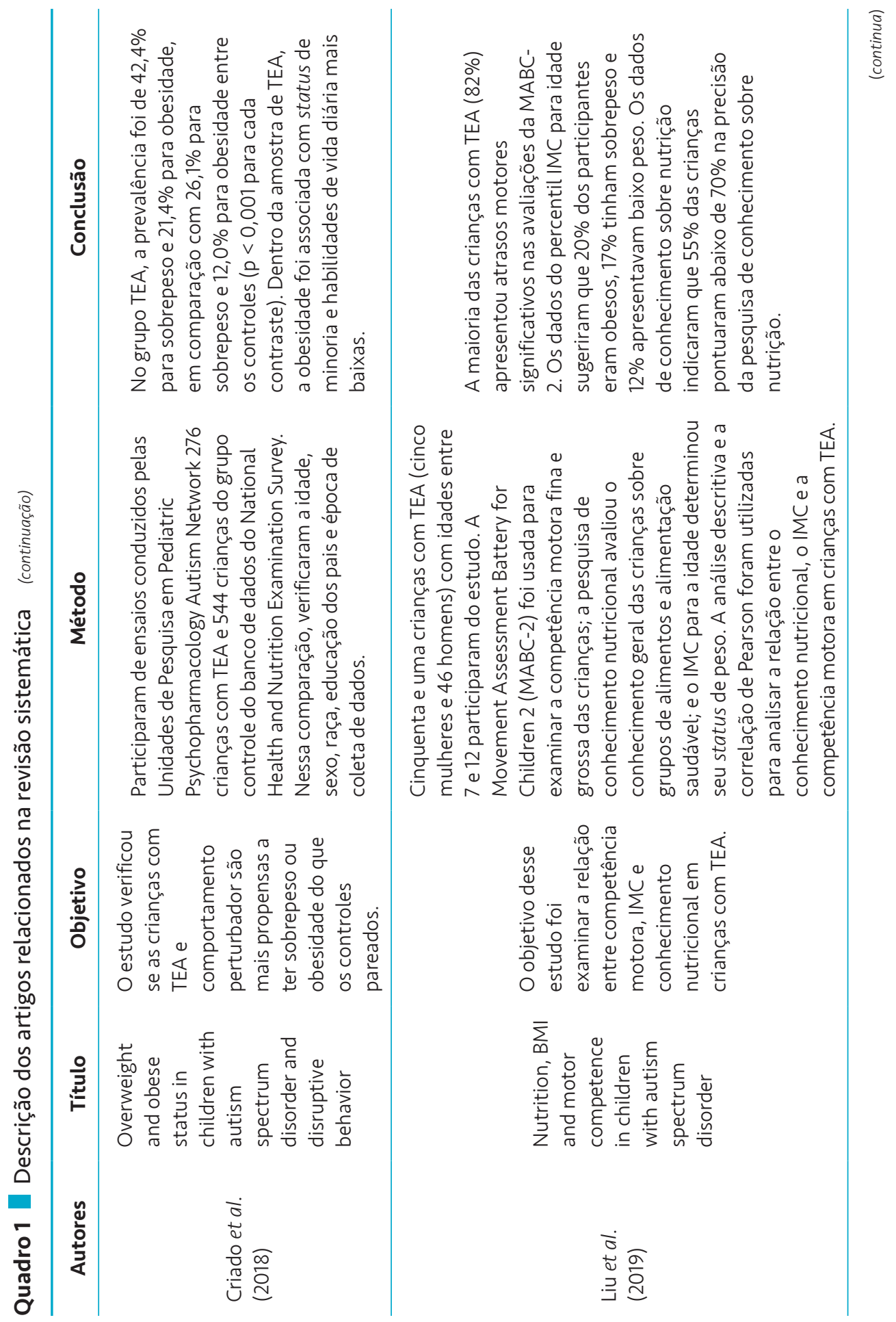




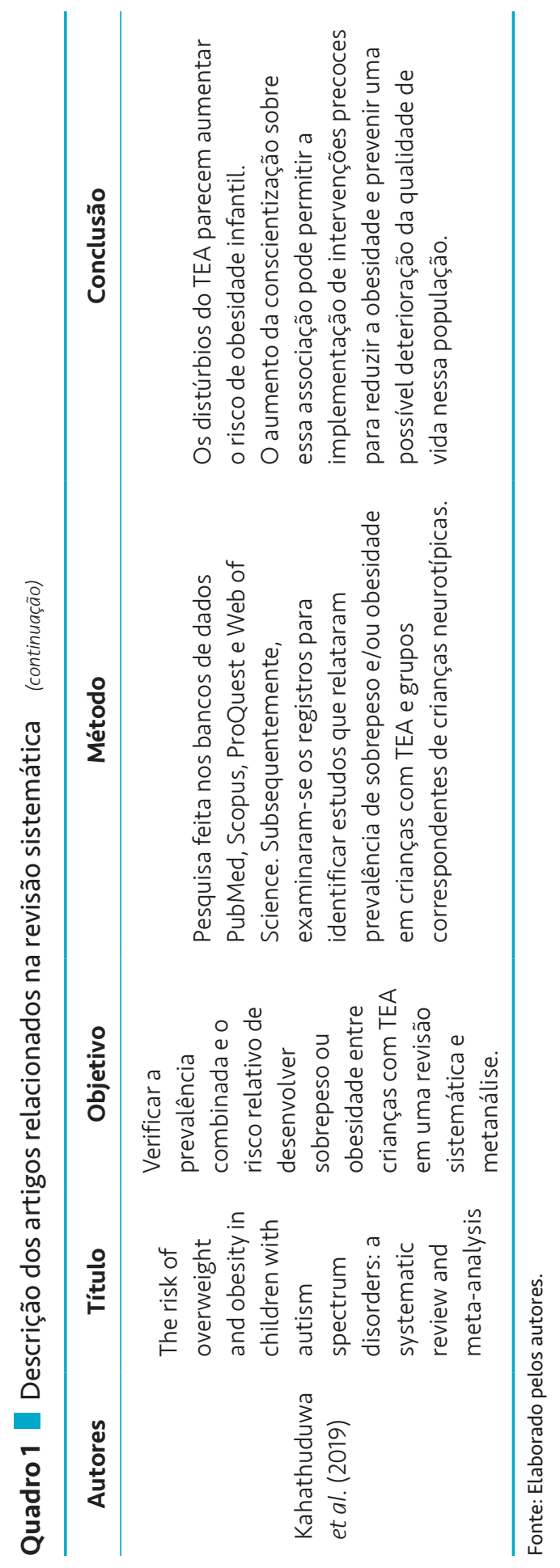


O Quadro 1 traz a síntese dos cinco artigos selecionados para este estudo, que usaram em seus procedimentos ferramentas de avaliação dos aspectos para identificar a prevalência da obesidade e do ganho excessivo de peso em indivíduos com TEA e suas causas.

De todos os artigos selecionados nesta pesquisa, não se encontrou nenhuma publicação em português, todos estão na língua inglesa. Apesar de os estudos apontarem a prevalência da obesidade e do sobrepeso em indivíduos com TEA e fatores que contribuem para essa prevalência alta, pode-se constatar que há uma escassez de trabalhos relacionados à obesidade e ao ganho excessivo de peso na população diagnosticada com autismo.

Dos artigos selecionados, um apresenta, em seus métodos, uma pesquisa bibliográfica com o objetivo de identificar fatores biológicos e comportamentais que possam contribuir para o ganho de peso não saudável em pessoas com TEA, além de fatores de risco (DHALIWAL et al., 2019).

Esse estudo aponta quatro fatores-chave que podem contribuir para o ganho não saudável de peso: comportamentos alimentares, estilo de vida, comorbidades e uso de medicamentos. A revisão mostra também evidências de que diversidade reduzida da microbiota intestinal, desequilíbrios hormonais e distúrbios metabólicos maternos podem influenciar a obesidade infantil. Além disso, o estudo destaca que ainda não está claro quanto ou em que medida esses fatores influenciam o ganho de peso. Nessa revisão, Dhaliwal et al. (2019) destacaram os seguintes fatores:

- Em algumas faixas etárias, constataram-se altas taxas de prevalência de comportamento alimentar, o que está ligado ao processamento sensorial oral anormal e ao comportamento de colocar tudo na boca, evitar certas texturas ou gostos ou apenas comer certos alimentos.

- Comportamento sedentário, falta de atividades físicas, desafios sociais e comportamentais e déficits motores são fatores-chave para que indivíduos com TEA não se engajem em programas de esportes.

De acordo com Dhaliwal et al. (2019), a genética também desempenha um papel na obesidade infantil, pois estudos apontam que irmãos ou pais obesos da pessoa com TEA têm maior risco de obesidade. Prescrições de medicações psicóticas para controlar algumas comorbidades do TEA, como transtorno de déficit de atenção e hiperatividade (TDAH) e depressão, podem 
contribuir para a obesidade. Uma metanálise realizada em 2016 mostrou que uma em cada seis crianças com TEA recebeu prescrições de antipsicóticos e medicamentos, como risperidona e aripiprazol, que com frequência são indicados para o controle de hiperatividade, irritabilidade e agressão. E já está comprovado que esses medicamentos estão associados ao ganho de peso.

Os autores também destacam fatores emergentes, como amamentação, sono e microbiota. Dhaliwal et al. (2019) relacionam alguns transtornos gastrointestinais comuns no TEA, como diarreia, constipação crônica e dores abdominais, e citam um estudo realizado com 163 alunos com TEA de uma escola, em que $25,8 \%$ dos participantes relataram ter pelo menos um sintoma gastrointestinal grave.

Dhaliwal et al. (2019) mencionam ainda influências endócrinas, como leptina, adiponectina, grelina e distúrbios metabólicos maternos, como fatores emergentes.

Segundo a revisão sistemática realizada por Kahathuduwa et al. (2019), os estudos analisados entre 1999 e 2018 incluíram crianças com idade entre 2 e 18 anos. Desses estudos, 21 realizaram pesquisas com crianças nascidas nos Estados Unidos, enquanto 16 mostraram dados de crianças que moravam em outros lugares do mundo. No que concerne à qualidade das evidências, dos 37 estudos analisados, 21 foram classificados como bons, e 16, como suplementares, de acordo com os critérios da National Institutes of Health.

Todos os estudos foram examinados com base nos requisitos da National Institutes of Health Study Quality Assessment Tool for Observational Cohort and Cross-Sectional Studies, e dois funcionários avaliaram cada estudo. As informações elegíveis foram disponibilizadas em uma planilha com dados predefinidos: título, autores, ano de publicação, periódico, número total de crianças com TEA, número de crianças com sobrepeso ou obesidade entre crianças com TEA, número total de crianças em desenvolvimento típico, número de crianças em desenvolvimento típico com sobrepeso ou obesidade, idade média, proporção de mulheres entre crianças com TEA, proporção de não caucasianos entre crianças com TEA, localização geográfica, média e quociente de inteligência (QI).

Os autores observaram que as estimativas de prevalência de sobrepeso e obesidade em crianças com TEA foram significativamente maiores do que as estimativas de prevalência populacional com mesmo sexo e faixa etária dentro dos Estados Unidos. O risco foi 41\% de os indivíduos desenvolverem sobrepeso. 
Apontaram também alguns fatores que podem contribuir para o sobrepeso e a obesidade, como depressão, medicamentos, diabetes e algumas comorbidades do TEA que afetam a qualidade de vida das pessoas diagnosticadas com TEA e aumentam ainda mais o custo do tratamento e das terapias.

Kahathuduwa et al. (2019) sugeriram outras revisões sistemáticas e metanálises referentes aos fatores que limitam os estudos sobre esse assunto, porque eles observaram que muitos estudos não apresentaram clareza em seus resultados. Essa ambiguidade foi relatada em diversas narrativas. Os autores relataram também que algumas revisões sistemáticas e uma metanálise que tentaram mostrar a relação entre obesidade e fatores que possam contribuir para o excesso de peso em pessoas com TEA usaram estudos tendenciosos.

O estudo de Nor, Ghozali e Ismail (2019) com 151 crianças com TEA entre 2 e 18 anos, realizado no Child Development Center at Universiti Kebangsaan Malaysia Medical Center, por meio de três questionários sobre sono, prática de atividades físicas e refeições, identificou uma prevalência de 11,3\% em crianças com sobrepeso e de $21,9 \%$ em crianças com obesidade. A idade média das crianças com sobrepeso e obesidade era de 8,5 anos.

Criado et al. (2018), em uma base com 276 crianças com TEA, compararam os níveis de obesidade e sobrepeso em crianças neurotípicas, e constataram o seguinte: prevalência de $42,4 \%$ para sobrepeso e $21,4 \%$ para obesidade, em comparação com $26,1 \%$ para sobrepeso e $12,0 \%$ para obesidade entre os neurotípicos. Os autores correlacionaram esse resultado com as habilidades de vida diária, pois os indivíduos com TEA pontuaram baixo nesse quesito.

Liu et al. (2019) correlacionam as questões de atrasos no desenvolvimento motor com sobrepeso e obesidade. Pode-se verificar no artigo que foram realizados testes motores específicos alinhados com a verificação do IMC e da qualidade de vida dos participantes. Os resultados demonstraram que, entre as crianças de 7 a 12 anos, 20\% eram obesas, 17\% tinham sobrepeso e $12 \%$ apresentavam baixo peso; a causa levantada no artigo foi a falta de conhecimento sobre nutrição e problemas nas competências motoras dos indivíduos com TEA.

O Quadro 2 apresenta os fatores fundamentais que influenciam a prevalência de obesidade e sobrepeso em pessoas com TEA. 
Quadro 2 Fatores que influenciam o sobrepeso e a obesidade no TEA

\begin{tabular}{c}
\hline Falta de conhecimento nutricional \\
\hline Inatividade física \\
\hline Medicamentos \\
\hline Transtornos mentais (ansiedade, depressão, entre outros) \\
\hline Competências motoras \\
\hline Distúrbios metabólicos
\end{tabular}

Fonte: Elaborado pelos autores.

Esses fatores não são muito diferentes dos observados em estudos de pessoas neurotípicas. Segundo a Organização Mundial da Saúde (2004), a obesidade é um dos dez maiores problemas de saúde pública. E no Brasil o cenário não muda, pois houve um grande aumento de pessoas obesas nas últimas três décadas. A obesidade está ligada a vários males, como alimentação irregular, diminuição da prática de esportes, entre outros. Com isso ela pode causar acidente vascular cerebral, doenças de articulações, depressão, câncer, entre outras enfermidades que afetam a qualidade de vida.

As pessoas com TEA, além do transtorno que interfere nos fatores de comunicação social, na seletividade alimentar, no sistema sensório-motor, nos interesses restritos e nos padrões repetitivos (AMERICAN PSYCHIATRIC ASSOCIATION, 2013), podem ter doenças crônicas, como diabetes e hipertensão. Nesse rol, pode-se incluir a obesidade, que prejudica ainda mais a qualidade de vida dos indivíduos com TEA.

\section{CONCLUSÃO}

Produções científicas sobre a prevalência do ganho excessivo de peso e da obesidade e sobre os fatores que podem contribuir para isso foram analisadas por este estudo qualitativo.

Constatou-se que os estudos selecionados para esta revisão bibliográfica corroboraram a necessidade de abordar essa temática, confirmando a escassez do tema na literatura científica. Por meio da análise realizada por este estudo, identificou-se que os indivíduos com TEA são mais suscetíveis a ter ganhos excessivos de peso e obesidade do que a população fora do espectro do autismo. Isso ocorre porque as pessoas autistas não praticam atividade física, não têm conhecimentos nutricionais, utilizam medicamentos específicos, entre outros fatores. 
Sugerem-se mais estudos sobre essa temática para que possam ser desenvolvidas políticas públicas mais eficazes no combate ao sobrepeso e à obesidade para a população com TEA.

\title{
Prevalence of overweight and obesity in people with autism spectrum disorder: a bibliographic review
}

\begin{abstract}
Obesity and excessive weight gain have been a public health problem in many countries and some researches point out that obesity is the second leading cause of preventable deaths in the United States, second only to smoking. Many studies point out that physical inactivity, deficits in social interaction, sedentary behavior, are related to the increased risk of obesity and excessive weight gain in people with autism spectrum disorder. The aim of this study was to survey and analyze articles on the prevalence and factors that may contribute to obesity, and weight gain in people with autism spectrum disorder through the PubMed and Bireme database. The method used was qualitative-descriptive, using specific descriptors autism and obesity and overweight and autism. There were 396 articles published between 2018 and 2020. Of this total, 5 were in the inclusion criteria of this study. The conclusion of this study was that individuals with autism have a higher risk of obesity and excessive weight gain in relation to the neurotypic population and several causes listed as physical inactivity and lack of nutritional knowledge are important factors for the installation of a condition that can cause problems chronic diseases and loss of quality of life.
\end{abstract}

\section{Keywords}

Autism. Obesity. Autism spectrum disorder. Overweight. Physical inactivity.

\section{Prevalencia de sobrepeso y obesidad en personas con trastorno del espectro autista: una revisión bibliográfica}

\section{Resumen}

La obesidad y el aumento de peso excesivo han sido un problema de salud pública en muchos países y algunas investigaciones señalan que la obesidad 
es la segunda causa principal de muertes evitables en los Estados Unidos, solo superada por el tabaquismo. Numerosos estudios señalan que la inactividad física, los déficits en la interacción social, el comportamiento sedentario, están relacionados con el mayor riesgo de obesidad y aumento de peso excesivo en personas con trastorno del espectro autista. El objetivo de este estudio fue relevar y analizar artículos sobre la prevalencia y los factores que pueden contribuir a la obesidad y al aumento de peso en personas con trastorno del espectro autista a través de la base de datos PubMed y Bireme. El método utilizado fue cualitativo-descriptivo, utilizando descriptores específicos autismo y obesidad, sobrepeso y autismo. Hubo 396 artículos publicados entre 2018 y 2020. De este total, 5 se encontraban en los criterios de inclusión de este estudio. La conclusión de este estudio fue que los individuos con autismo tienen un mayor riesgo de obesidad y aumento de peso excesivo en relación a la población neurotípica y varias causas enumeradas como inactividad física y falta de conocimiento nutricional son factores importantes para la instalación de una condición que puede causar problemas y enfermedades crónicas y pérdida de la calidad de vida.

\section{Palabras clave}

Autismo. Obesidad. Trastorno del espectro autista. Sobrepeso. Inactividad física.

\section{REFERÊNCIAS}

ABREU, L. C. de. Condições relacionadas à obesidade secundária na interface do crescimento e desenvolvimento. Revista Brasileira de Crescimento e Desenvolvimento Humano, São Paulo, v. 21, n. 1, p. 7-10, 2011. Disponível em: http://pepsic.bvsalud.org/ scielo.php? script $=$ sci_arttext\&pid $=$ S0104-12822011000100001\&lng $=$ pt\&nrm $=$ iso. Acesso em: 17 ago. 2020.

AMERICAN PSYCHIATRIC ASSOCIATION. Diagnostic and statistical manual of mental disorders: DSM-5. Arlington, VA: APA, 2013.

BLOCK, M. E. A teacher's guide to adapted physical educational: including students with disabilities in sports and recreation. 4. ed. Baltimore: Brookes, 2016.

CRIADO, K. K. et al. Overweight and obese status in children with autism spectrum disorder and disruptive behavior. Autism, v. 22, n. 4, p. 450-459, 2018. DOI: $10.1177 / 1362361316683888$

DHALIWAL, K. K. et al. Risk factors for unhealthy weight gain and obesity among children with autism spectrum disorder. International Journal of Molecular Sciences, v. 20 , n. 13, p. 1-2, 2019. 
KAHATHUDUWA, C. N. et al. The risk of overweight and obesity in children with autism spectrum disorders: a systematic review and meta-analysis. Obesity Reviews, v. 20, n. 12, p. 1667-1670, 2019. DOI: 10.1111/obr.12933

KUMMER, A. et al. Frequência de sobrepeso e obesidade em crianças e adolescentes com autismo e transtorno do déficit de atenção/hiperatividade. Revista Paulista de Pediatria, v. 34, n. 1, p. 71-77, 2016. DOI: 10.1016/j.rpped.2015.06.013

LIU, T. et al. Nutrition, BMI and motor competence in children with autism spectrum disorder. Medicina, v. 55, n. 5, p. 1-12, 2019. DOI:10.3390/medicina55050135

NOR, N. K.; GHOZALI, A. H.; ISMAIL, J. Prevalence of overweight and obesity among children and adolescents with autism spectrum disorder and associated risk factors. Frontiers in Pediatrics, v. 7, p. 38, 2019. DOI:10.3389/fped.2019.00038

ORGANIZAÇÃO MUNDIAL DA SAÚDE. Obesidade: prevenindo e controlando a epidemia global. Relatório da consultoria da OMS. Tradução Andréa Favano; revisão científica Sérgio Setsuo Maeda. São Paulo: Roca, 2004. 